\title{
NEURAL STEM CELLS TRANSPLANTED INTO INTACT BRAINS AS NEUROSPHERES FORM SOLID GRAFTS COMPOSED OF NEURONS, ASTROCYTES AND OLIGODENDROCYTE PRECURSORS
}

\author{
Jana Karbanová*, Jaroslav Mokrý, Lenka Kotingová
}

Department of Histology and Embryology, Charles University in Prague, Faculty of Medicine in Hradec Králové, Šimkova 870 , P. O. Box 38, 50038 Hradec Králové, Czech Republic

e-mail: karbanovaj@lfhk.cuni.cz

Received: September 20, 2004

Key words: Neural stem cells/Neurospheres/Rat/Transplantation/Differentiation/Nestin

Neural stem cells (NSCs) are tissue-specific stem cells with self-renewal potential that can give rise to neurons and glia in vivo and in vitro. The aim of this study was to transplant NSCs as whole neurospheres into intact brain and assess the fate and phenotype of their progeny generated in vivo. We isolated NSCs from E14 foetal rat forebrains and cultured them in basic fibroblast and epidermal growth factor-supplemented serum-free medium in the form of neurospheres in vitro. Neurospheres were transplanted into the intact brains of 2 Wistar rats and after a period of 3 weeks, grafted brains were examined immunohistochemically. Neurospheres formed solid grafts that were found in the lateral ventricle and in the velum interpositum under the hippocampus. The majority of cells in the transplanted tissue were identified as $\beta$-III-tubulin ${ }^{+}, \mathrm{NeuN}^{+}, \mathrm{PanNF}^{+}$and synaptophysin ${ }^{+}$neurons and were accumulated throughout the graft centre. $\mathrm{GFAP}^{+}$astrocytes were scattered throughout the entire graft and astrocyte processes delimited the outer and perivascular surfaces. A great number of $\mathrm{NG}_{2}{ }^{+}$oligodendrocyte precursors was detected. Nestin ${ }^{+}$endothelial cells were found to line capillaries growing in the transplant. These data indicate that nestin ${ }^{+}$NSCs prevailing in neurospheres differentiate following transplantation into nestin neuronal and glial cells which confirms the multipotency of NSCs. Three weeks posttranpslantation neuronal and astrocyte cells reached terminal differentiation (formation of synaptic vesicles and superficial and perivascular limiting membranes) while elements of oligodendroglial cell lineage remained immature. Grafting stem cells as non-dissociated neurospheres provide cells with favourable conditions which facilitate cell survival, proliferation and differentiation. However, in the intact brain, grafted neurosphere cells were not found to integrate with the brain parenchyma and formed a compact structure demarcated from its surroundings.

\section{INTRODUCTION}

A number of studies have analysed the origin and development of neural stem cells, which are clonogenic cells with self-renewal and multipotency. Cultured stem cells represent a potential cell source for transplantation and a useful model for developmental studies. Neural stem cells have been isolated from embryonic ${ }^{1,2}$ and adult brains $^{3}$. They are identified by staining for the intermediate filament nestin ${ }^{4}$. In culture, stem cells grow in suspension as spherical aggregates (neurospheres) in defined medium supplemented with EGF and/or bFGF ${ }^{1,5,6}$. Neurospheres contain multipotent stem cells that are normally represented in very small numbers and progenitor cells that are more restricted in their proliferative and phenotypic potentials and are derived from stem cells. Neurospheres may be dissociated and subcultured repeatedly. Plating them on adhesive substrate induces phenotypic differentiation into neurons, astrocytes, and oligodendrocytes, preceded by migration of cells out of the neurospheres. The aim of this study was to transplant neural stem cells as nondissociated neurospheres and examine their ability to survive and differentiate in vivo.

\section{MATERIAL AND METHODS}

NSCs isolation and culture. Neural stem cells were isolated from E14 foetuses of timed pregnant Wistar rats. Foetal forebrains were dissected free of meninges and mechanically dissociated by trituration through a fire polished pipette. Single cell suspension of neural progenitors was plated into the culture flasks with untreated surfaces $\left(40,000 / \mathrm{cm}^{2}\right)$. The culture medium was composed of Dulbecco's Modified Eagle's Medium / Ham's F12 (1:1; Sigma), B27 Supplement (Gibco), 2 mM L-Glutamine (Sigma), $100 \mathrm{U} / \mathrm{ml}$ penicillin and $100 \mu \mathrm{g} / \mathrm{ml}$ streptomycin (Gibco), $10 \mathrm{ng} / \mathrm{ml}$ basic fibroblast growth factor (bFGF, PeproTech) and $20 \mathrm{ng} / \mathrm{ml}$ epidermal growth factor (EGF, PeproTech). Neural stem cells grew in culture as free-floating neurospheres and fresh growth factors were added to the medium every $3^{\text {rd }}$ day. For the experiment, neurospheres cultured for 6 days in vitro were used.

Transplantation. Two adult Wistar female rats (VELAZ, Prague, Czech Republic) approximately 3 months old and weighing 180-200 g were used in this study. Prior to surgery, rats were anaesthetized with a $1.5 \mathrm{~mL} / \mathrm{kg}$ intraperitoneal injection of a solution containing $5 \mathrm{mg} / \mathrm{mL}$ xy- 
lazine and $25 \mathrm{mg} / \mathrm{mL}$ ketamine. Under aseptic conditions, a midline incision was made in the scalp and the sagittal and coronal sutures were identified. A small craniotomy was made with the aid of a burr-drill above the right cerebral hemisphere $2 \mathrm{~mm}$ anterior from bregma and $1.5 \mathrm{~mm}$ lateral from the midline. Neurospheres that were removed from the medium and rinsed with PBS were transplanted unilaterally 4-5 $\mathrm{mm}$ in an amount of $2 \times 10^{6} / 10 \mu 1$ deep in the right cerebral brain hemisphere. After the needle was withdrawn, the incision was closed with a suture.

Histology. Animals were sacrificed at 3 weeks following transplantation. Under deep anaesthesia, they were transcardially perfused with $10 \%$ formalin. Brains were removed and immersed in the same fixative for at least three days. Neurospheres were collected from cultivation medium by centrifugation, rinsed with PBS and fixed in $10 \%$ formalin for 1 hour. Brains or neurospheres were dehydrated in increasing concentrations of alcohols and embedded in paraffin. Serial seven-micrometre thick neurosphere sections or coronal brain sections were mounted on glass slides. Brain sections containing grafted cells or neurosphere sections were processed for a variety of immunohistochemical detections to allow identification of differentiated cell types.

Immunohistochemistry. Deparaffinised and rehydrated sections were incubated for $20 \mathrm{~min}$ in methanol containing $1 \% \mathrm{H}_{2} \mathrm{O}_{2}$ to reduce endogenous peroxidase activity. Then, sections were exposed to microwaves $(700 \mathrm{~W})$ in sodium citrate solution for 2 x $5 \mathrm{~min}$ (antigen retrieval). After thorough washing in $0.2 \mathrm{M}$ Tris- $\mathrm{HCl}$ buffer containing $0.5 \%$ Triton X-100 (Sigma), sections were incubated with a primary antibody for $45 \mathrm{~min}$. Mouse monoclonal antibodies were utilised for the detection of nestin (clone rat401, Developmental Studies Hybridoma Bank (DSHB), Iowa City IA, USA, 1:4), GFAP (clone GA-P, Sigma, $1: 400), \beta$-III-tubulin (clone Tuj-1, RD systems); NeuN (clone A60, Chemicon, 1:100); PanNF (clone DA2, FNP7, RmdO20.11; Zymed; 1: 100), synaptophysin (clone SY 38, Dako, 1:25), O4 (clone 81, Boehringer, 1:5) and MBP (clone 130-137, Sigma, 1:40). Polyclonal antibody was used to identify NG2 (Chemicon, 1:200). Antibody purchased from the DSHB was obtained under contract N01-HD-6-2915 from the NICHD. Following washing, sections were incubated with a species-specific secondary biotinylated antibody (goat anti-mouse IgG or goat antirabbit IgG; Sigma) for $45 \mathrm{~min}$ and then with streptavidin conjugated with horseradish peroxidase (BioGenex). After rinsing, the peroxidase activity was visualised using 3,3'-diaminobenzidine tetrahydrochloride (Sigma) and hydrogen peroxide. Sections were counterstained with methyl green, dehydrated and mounted in DPX (Sigma). To avoid false positivity, parallel sections were processed according to the same protocol but primary antibodies were omitted. Slides were examined with an Olympus BX50 microscope.

\section{RESULTS AND DISCUSSION}

Neural stem cells isolated from foetal forebrains proliferated in response to growth factors in culture medium and formed neurospheres. After six days in vitro, these cell clusters measuring about $60-80 \mu \mathrm{m}$ in diameter consisting of several hundred cells were transplanted in intact rat brains. Immunohistochemical analysis of neurospheres yielded from culture proved that nestin was in the the majority of cells and was localized mainly in the surface layers. Neurosphere cells were negative for GFAP and $\beta$ III-tubulin, markers of differentiated neural cells. Central parts of neurospheres showed lower cellular density and large intercellular spaces. Some apoptotic and necrotic cells were found in the central areas. Three weeks after neurosphere transplantation, the graft was identified in coronal brain sections as a solid irregularly rounded structure in the right lateral ventricle or as an oval elongated structure between the lateral and third ventricles under the hippocampus. In haematoxylin-eosin this grafted tissue stained more lightly than the neighbouring parenchyma. The central part of the transplant contained accumulated large pale-stained cell nuclei, which were identified immunohistochemically as $\mathrm{NeuN}^{+}$nuclei of neurons (Fig. A). The periphery of the graft was devoid of neurons. A small number of neurons revealed positivity for PanNF. Weak immunopositivity for neurofilaments was detected in the cell body and strong positivity was expressed in the neuronal processes (Fig. C). Immunohistochemical detection of $\beta$-III-tubulin showed strong positivity mainly in the axon and dendrite processes on which the dendrite spines were visible. Weak $\beta$-III-tubulin-positivity was detected in the cytoplasma around the cell nuclei (Fig. E). Immunoreactivity for synaptophysin, a glycoprotein found in synaptic vesicles detected in the vicinity neuronal pericarya, indicated that neuronal cells underwent terminal differentiation. Multipotent neural stem cells gave also rise to glial cells. Astrocytes were identified using the GFAP antibody as stellate cells with short processes (Fig. B). These cells were distributed irregularly in the entire transplant with the higher cell density at the graft periphery. These peripheral astrocytes lined the graft surface by the flattened end-feet of processes forming a superficial limiting membrane. A similar limiting membrane was observed around blood capillaries. Immunodetection of NG2 revealed the wide ramifying processes of oligodendrocyte precursors with intense small cell bodies (Fig. D). Nevertheless, transplanted cells were immunonegative for $\mathrm{O} 4$ and MBP. Immunohistochemical detection of intermediate filament nestin showed positivity only in the flattened endothelial cells lining the small blood vessels inside the graft and in the adjacent parenchyma.

This study is the first report on transplantation of nondissociated rat neurospheres into the intact non-injured brain. The collected data demonstrate that neural stem cells transplanted into intact brains as neurospheres can survive, expand and differentiate into $\mathrm{NeuN}^{+}, \beta$-IIItubulin $^{+}$and PanNF$^{+}$neurons, $\mathrm{GFAP}^{+}$astrocytes and im- 

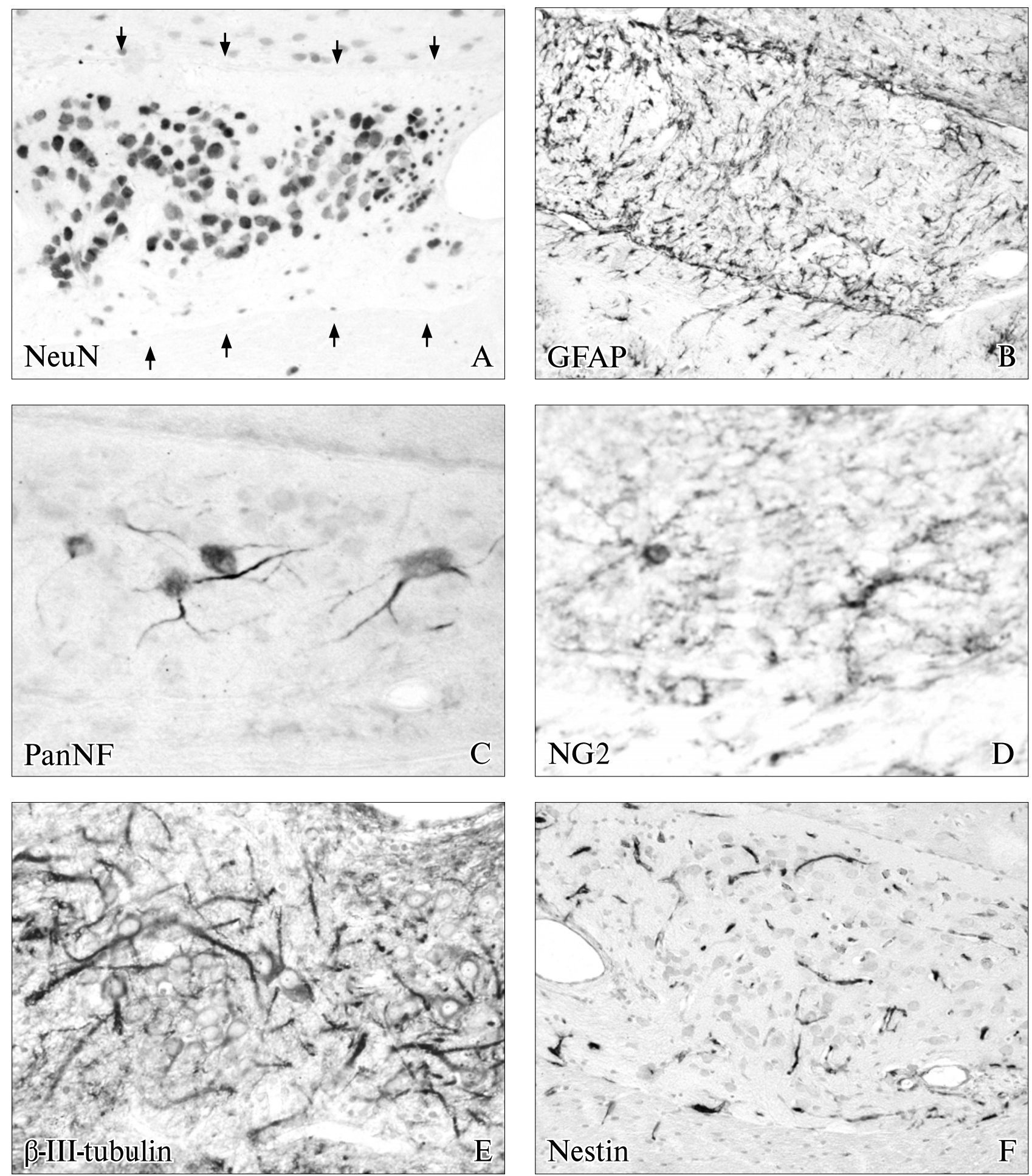

Fig. 1. A-F: Photomicrographs of immunohistochemical detections of grafted neurospheres into the rat brain 3 weeks posttransplantation. Scale bars: $50 \mu \mathrm{m}$.

A, C, E: Neurosphere cells differentiated mainly into neurons immunopositive for NeuN (A), but not all NeuN ${ }^{+}$ neurons were PanNF (C) and $\beta$-III-tubulin (E). Neuronal cells bodies were located in the centre of the graft (Fig. A); the graft margins are marked with arrows.

B, D: Transplanted neural stem cells differentiated also into glial cells. GFAP ${ }^{+}$astrocytes were scattered in the whole grafted tissue and processes of surface astrocytes formed the superficial limiting membrane. Oligodendrocyte precursors were visualized using NG2 antibody that binds to chodroitin sulfate proteoglycans on the cell membrane surface.

F: Nestin positivity was detected in endothelial cells of small blood vessels in the transplant and adjacent brain parenchyma. 
mature $\mathrm{NG}^{+}$oligodendrocyte precursors. Neurospheres after transplantation aggregated, expanded and merged into solid formation, which spread into easily accessible spaces between brain structures. Grafts that were found in the lateral cerebral ventricle or in the space between the lateral and third ventricles just under the hippocampus were strictly delimited from surrounding tissue by the membrana limitans gliae superficialis on both the graft and adjacent brain tissue. However, we could not exclude single cells migration from transplant to surrounding tissue because we did not use for transplantation labelled cells. The graft was connected with neighbouring tissue via blood vessels that penetrated the transplant providing nourishment of transplanted cells. The endothelium of capillaries expressed nestin positivity which indicated that these vessels were newly formed ones ${ }^{7}$. Although neurospheres cultured 6 days in vitro contained large numbers of stem cells expressing nestin, 3 weeks following transplantation, none of the transplanted cells revealed nestin positivity. This finding provides evidence that transplanted neurospheres were composed of immature stem or progenitor cells that following transplantation gave rise to three major CNS cell types. This confirms the multipotency of stem cells, the principal property of stem cells. Neurons reached terminal differentiation as documented by their ability to form synaptic vesicles. Astrocytes also underwent maximal specialization by forming superficial and perivascular limiting membranes; the latter is necessary for the induction of the blood brain barrier. Even though mature oligodendrocytes were not detected it can be anticipated that over a longer time period they can maturate morphologically and functionally into mature oligodendrocytes. This supports results from our experiments with neurosphere differentiation in vitro, in which oligodendrocytes are the last cell type arising from stem cells during the third week. It is not clear whether neurosphere cells differentiated and expanded under the influence of local cues produced by adjacent cells as is known in the case of injured brain tissue ${ }^{8}$. What was surprising however was that the size of the transplant was much larger than the volume of transplanted cells. Transplantation of the whole neurosphere probably enables it to maintain the original cell-cell contacts established during neurosphere formation, which is necessary for survival and proliferation in vitro ${ }^{9}$. It is likely that neurosphere cells secrete factors that encourage survival and proliferation for short periods following transplantation ${ }^{10}$ and compensate the lack of mitogens after withdrawal of cell from cultured medium.

\section{ACKNOWLEDGEMENTS}

The authors are grateful to Mrs. Helena Rückerová and Mrs. Hana Hollerová for their skilful technical assistance. The work was supported by grant number 304/03/1515 from the Grant Agency of the Czech Republic.

\section{REFERENCES}

1. Reynolds BA, Tetzlaff W, Weiss S. (1992) A multipotent EGF responsive striatal embryonic progenitor cell produces neurons and astrocytes. J Neurosci 12, 4565-74.

2. Davis AA, Temple S. (1994) A self-renewing multipotential stem cell in embryonic rat cerebral cortex. Nature 372, 263-6.

3. Reynolds BA, Weiss S. (1992) Generation of neurons and astrocytes from isolated cells of the adult mammalian central nervous system. Science 255, 1707-10.

4. Lendahl U, Zimmermann LB, McKay RDG. (1990) CNS stem cells express a new class of intermediate filament protein. Cell 60 , 585-95.

5. Vescovi AL, Reynolds BA, Fraser DD, Weiss S. (1993) bFGF regulates the proliferative fate of unipotent (neuronal) and bipotent (neuronal/astroglial) EGF-generated CNS progenitor cells. Neuron 11, 951-66.

6. Gritti A, Parati EA, Cova L, Frölichsthal P, Galli R, Wanke E, Faravelli L. (1996) Multipotent stem cells from the adult mouse brain proliferate and self-renew in response to basic fibroblast growth factor. J Neurosci 16, 1091-100.

7. Mokrý J, Němeček S. (1999) Cerebral angiogenesis shows nestin expression in endothelial cells. Gen Physiol Biophys 18 (Suppl. 1), $25-9$.

8. Wennersten A, Meijer X, Holmin S, Wahlberg L, Mathiesen T. (2004) Proliferation, migration, and differentiation of human neural stem/progenitor cells after transplantation into a rat model of traumatic brain injury. J Neurosurg 100, 88-96.

9. Svendsen $\mathrm{CN}$, ter Borg MG, Armstrong RJE, Rosser AE, Chandran S, Ostenfeld T, Caldwell CA. (1998) A new method for the rapid and long term growth of human neural precursor cells. J Neurosci Methods 85, 141-52.

10. Burnstain, RM, Foltynie T, He X, Menon, DK, Svendsen CN, Caldwell MA. (2004) Differentiation and migration of long term expanded human neural progenitors in a partial lesion model of Parkinson's disease. Int J Biochem Cell Biol 36, 702-13. 